\title{
EVIDENCE FOR UBIQUITOUS HIGH-EQUIVALENT-WIDTH NEBULAR EMISSION IN $z \sim 7$ GALAXIES: TOWARD A CLEAN MEASUREMENT OF THE SPECIFIC STAR-FORMATION RATE USING A SAMPLE OF BRIGHT, MAGNIFIED GALAXIES
}

\author{
R. Smit ${ }^{1}$, R. J. Boumens ${ }^{1}$, I. Labbé ${ }^{1}$, W. Zheng ${ }^{2}$, L. Bradley ${ }^{3}$, M. Donahue ${ }^{4}$, D. Lemze ${ }^{2}$, J. Moustakas ${ }^{5}$, K. Umetsu $^{6}$, \\ A. Zitrin ${ }^{7,8,24}$, D. $\mathrm{CoE}^{3}$, M. Postman ${ }^{3}$, V. GonZalez ${ }^{9}$, M. Bartelmann ${ }^{7}$, N. Benítez ${ }^{10}$, T. Broadhurst ${ }^{11}$, H. Ford $^{2}$,

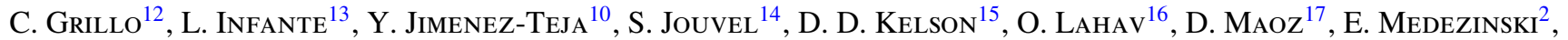 \\ P. Melchior ${ }^{18}$, M. Meneghetti ${ }^{19}$, J. Merten ${ }^{20}$, A. Molino ${ }^{10}$, L. A. MoustaKas ${ }^{20}$, M. Nonino ${ }^{21}$, P. Rosati ${ }^{22}$, And S. Seitz ${ }^{23}$ \\ ${ }^{1}$ Leiden Observatory, Leiden University, NL-2300 RA Leiden, The Netherlands \\ ${ }^{2}$ Department of Physics and Astronomy, The Johns Hopkins University, 3400 North Charles Street, Baltimore, MD 21218, USA \\ ${ }^{3}$ Space Telescope Science Institute, 3700 San Martin Drive, Baltimore, MD 21208, USA \\ ${ }^{4}$ Department of Physics and Astronomy, Michigan State University, East Lansing, MI 48824, USA \\ ${ }^{5}$ Siena College, 515 Loudon Road, Loudonville, NY 12211, USA \\ ${ }^{6}$ Institute of Astronomy and Astrophysics, Academia Sinica, P. O. Box 23-141, Taipei 10617, Taiwan \\ ${ }^{7}$ Institut fur Theoretische Astrophysik, ZAH, Albert-Ueberle-Straß e 2, 69120 Heidelberg, Germany \\ ${ }^{8}$ Cahill Center for Astronomy and Astrophysics, California Institute of \\ Technology, MS 249-17, Pasadena, CA 91125, USA \\ 9 Department of Physics and Astronomy, University of California, Riverside, CA 92521, USA \\ ${ }^{10}$ Instituto de Astrofisica de Andalucia (CSIC), C/Camino Bajo de Huetor 24, Granada 18008, Spain \\ ${ }^{11}$ Department of Theoretical Physics, University of the Basque Country, P. O. Box 644, 48080 Bilbao, Spain \\ ${ }^{12}$ Dark Cosmology Centre, Niels Bohr Institute, University of Copenhagen, \\ Juliane Maries Vej 30, DK-2100 Copenhagen, Denmark \\ ${ }^{13}$ Departamento de Astronoia y Astrofisica, Pontificia Universidad Catolica de Chile, V. Mackenna 4860, Santiago 22, Chile \\ ${ }^{14}$ IInstitut de Cincies de l'Espai (IEEC-CSIC), Bellaterra (Barcelona), Spain \\ 15 Observatories of the Carnegie Institution of Washington, Pasadena, CA 91 101, USA \\ ${ }^{16}$ Department of Physics \& Astronomy. University College London, Gower Street, London WCIE 6 BT, UK \\ ${ }^{17}$ School of Physics and Astronomy, Tel Aviv University, Tel-Aviv 69978, Israel \\ ${ }^{18}$ Center for Cosmology and Astro-Particle Physics, \& Department of Physics; The Ohio State University, \\ 191 W. Woodruff Ave., Columbus, Ohio 43210, USA \\ ${ }^{19}$ INAF, Osservatorio Astronomico di Bologna, \& INFN, Sezione di Bologna, Via Ranzani 1, I-40127 Bologna, Italy \\ 20 Jet Propulsion Laboratory, California Institute of Technology, MS 169-327, Pasadena, CA 91109, USA \\ ${ }^{21}$ INAF-Osservatorio Astronomico di Trieste, via G.B. Tiepolo 11, 40131 Trieste, Italy \\ ${ }^{22}$ ESO-European Southern Observatory, D-85748 Garching bei Munchen, Germany \\ ${ }^{23}$ Universitats-Sternwarte, Munchen, Scheinerstr. 1, D-81679 Munchen. Germany \\ Received 2013 July 22; accepted 2014 January 17; published 2014 March 4
}

\begin{abstract}
Growing observational evidence indicates that nebular line emission has a significant impact on the rest-frame optical fluxes of $z \sim 5-7$ galaxies. This line emission makes $z \sim 5-7$ galaxies appear more massive, with lower specific star-formation rates (sSFRs). However, corrections for this line emission have been difficult to perform reliably because of huge uncertainties on the strength of such emission at $z \gtrsim 5.5$. In this paper, we present the most direct observational evidence thus far for ubiquitous high-equivalent-width (EW) [O III] $+\mathrm{H} \beta$ line emission in Lyman-break galaxies at $z \sim 7$, and we present a strategy for an improved measurement of the sSFR at $z \sim 7$. We accomplish this through the selection of bright galaxies in the narrow redshift window $z \sim 6.6-7.0$ where the Spitzer/Infrared Array Camera (IRAC) $4.5 \mu \mathrm{m}$ flux provides a clean measurement of the stellar continuum light, in contrast with the $3.6 \mu \mathrm{m}$ flux, which is contaminated by the prominent $[\mathrm{O} \mathrm{III}]+\mathrm{H} \beta$ lines. To ensure a high signal-to-noise ratio for our IRAC flux measurements, we consider only the brightest $\left(H_{160}<26\right.$ mag $)$ magnified galaxies we have identified behind galaxy clusters. It is remarkable that the mean rest-frame optical color for our bright seven-source sample is very blue, [3.6]-[4.5] $=-0.9 \pm 0.3$. Such blue colors cannot be explained by the stellar continuum light and require that the rest-frame EW of [O III] $+\mathrm{H} \beta$ is greater than $637 \AA$ for the average source. The four bluest sources from our seven-source sample require an even more extreme EW of $1582 \AA$. We can also set a robust lower limit of $\gtrsim 4 \mathrm{Gyr}^{-1}$ on the sSFR of our sample based on the mean spectral energy distribution.
\end{abstract}

Key words: galaxies: evolution - galaxies: formation - galaxies: high-redshift

Online-only material: color figures

\section{INTRODUCTION}

In the past decade, the evolution of galaxies at the earliest times has been predominantly mapped out by studying the restframe ultraviolet (UV) light in galaxies across cosmic time (e.g., Stanway et al. 2003; Bouwens et al. 2007, 2011; Lorenzoni et al. 2011; Oesch et al. 2012, 2013; Bradley et al. 2012b; Bowler et al. 2012; Schenker et al. 2013b). Despite great progress in

\footnotetext{
${ }^{24}$ Hubble Fellow.
}

this area, an equally important part of the story involves the buildup of mass in galaxies and the specific star-formation rate (sSFR, i.e., the SFR divided by the stellar mass), which provide direct constraints on the growth time scale of individual sources (Stark et al. 2009; González et al. 2010). Typical sSFRs of starforming galaxies at $z \sim 2\left(M_{*} \sim 5 \times 10^{9} M_{\odot}\right)$ are $\sim 2 \mathrm{Gyr}^{-1}$, equivalent to a doubling time of $\sim 500 \mathrm{Myr}$.

Over the past few years, substantial improvements have been made in how we characterize the sSFR in high-redshift galaxies 
and how the sSFR evolves. Initial observational studies found little evolution in the sSFR from $z \sim 2$ to higher redshift in apparent disagreement with theories of star formation fueled by cold accretion (Stark et al. 2009; González et al. 2010; Labbé et al. 2010a, 2010b; Weinmann et al. 2011). However, the effect of nebular emission lines (e.g., [O III], [O II], $\mathrm{H} \alpha$ ) that can contaminate the Infrared Array Camera (IRAC) measurements of the stellar continuum light had not been taken into account (e.g., Schaerer \& de Barros 2009, 2010).

The effect of this emission on broadband IRAC measurements can be considerable. Extrapolating the $\mathrm{H} \alpha$ equivalent widths (EWs) measured by Fumagalli et al. (2012) and Erb et al. (2006) to higher redshifts suggests $\mathrm{H} \alpha \mathrm{EWs}$ as large as $1000 \AA$ at $z \gtrsim 6$. This would indicate that $\sim 45 \%$ of the flux in [4.5] is due to $\mathrm{H} \alpha$ for galaxies at $z \sim 6-7$, while $[\mathrm{O} \mathrm{III}]+\mathrm{H} \beta$ can contribute $\sim 55 \%$ of the flux in [3.6]. Correcting for the effects of nebular emission, one can derive sSFRs that are plausibly consistent with theoretical expectations (Stark et al. 2013; González et al. 2014; de Barros et al. 2012).

As the previous discussion indicates, it is essential in quantifying the sSFR at $z>5$ to characterize the EWs of nebular emission lines and their impact on the IRAC photometry. Pioneering studies in the past two years have quantified the strength of nebular emission lines at $z \gtrsim 4$, through the measured flux offsets to the Spitzer/IRAC [3.6] and [4.5] bands. Shim et al. (2011) compare the [3.6] and [4.5] fluxes at $z \sim 4$ and show that the [3.6]-[4.5] color correlates with the SFR, implying that the source of the offset is likely due to the presence of $\mathrm{H} \alpha$ emission lines. Stark et al. (2013) estimate the influence of $\mathrm{H} \alpha$ on the [3.6] flux at $z \sim 3-4$ by comparing the color distribution of contaminated and uncontaminated spectroscopic confirmed galaxies (see also Schenker et al. 2013a) and extrapolating the observed emission line contamination to $z \sim 5-7$.

The first attempt to derive nebular line EWs for a large sample of Lyman-break galaxies (LBGs) at $z \gtrsim 5$ is presented in Labbé et al. (2013), based on a comparison of a stacked [3.6] and [4.5] flux measurement at $z \sim 8$ from the IRAC Ultra Deep Field program with similar flux measurements from a stacked sample at $z \sim 7$ (see also González et al. 2012 who make inferences about the EWs of nebular emission lines from the stacks of $z \sim 4-6$ galaxies). Estimates of the nebular-line EWs have also been made from direct fits to large number of spectroscopically confirmed $z \sim 4-7$ galaxies (de Barros et al. 2012; Ono et al. 2012; Tilvi et al. 2013; Curtis-Lake et al. 2013).

Even making use of the aforementioned methods, the sSFR in $z \sim 6-8$ galaxies is still very uncertain. Although one can certainly estimate the sSFR in this redshift range by using an extrapolation of the $\mathrm{H} \alpha$ EWs found at $z \sim 4$ to higher redshift, extrapolations are inherently uncertain. Results on the sSFR at $z \sim 8$ (Labbé et al. 2013), although providing good leverage to constrain the redshift evolution, are limited by the extreme faintness of the individual galaxies whose redshift distribution is only approximately known. Finally, the typical $\mathrm{H} \alpha \mathrm{EW}$ in $z \sim 4$ galaxies used for sSFR estimates has been established primarily through sources which show Ly $\alpha$ in emission; however, it is unclear whether those sources are representative of the broader $z \sim 4$ population (for more discussion, see Schenker et al. 2013a).

To overcome these issues, here we make use of a new strategy for measuring the sSFRs and stellar masses for galaxies at very high redshifts, while simultaneously obtaining very good constraints on the EWs of [O III] $+\mathrm{H} \beta$ line emission. Our plan is to take advantage of the considerable quantity of deep, wide-area observations over the 524 orbit, 25 cluster Cluster Lensing And Supernova survey with Hubble (CLASH) program (Postman et al. 2012) and other programs observing strong lensing clusters with deep multiband Hubble Space Telescope (HST) data. We select a small sample of bright, magnified galaxies for which we can obtain a clean measurement of the stellar continuum light from the deep IRAC observations over these clusters. One particularly fruitful redshift window in which we can obtain such clean measurements is the redshift window $z \sim 6.6-7.0$, where [4.5] is completely free of any emission lines. This should allow us to place much more robust constraints on the SSFR and the EW of nebular emission of star-forming galaxies at $z \sim 7$.

This paper is organized as follows. In Section 2, we discuss our data set, our photometric procedure, and source selection. In Section 3, we present the properties of our selected $z \sim 7$ sample. We discuss the constraints we put on the EWs of $\mathrm{H} \alpha, \mathrm{H} \beta$ and $[\mathrm{O}$ III] and the sSFR. We present a summary and discussion of our results in Section 4.

Throughout this paper, we adopt a Salpeter initial mass function (IMF) with limits $0.1-100 M_{\odot}$ (Salpeter 1955). For ease of comparison with previous studies, we take $H_{0}=$ $70 \mathrm{~km} \mathrm{~s}^{-1} \mathrm{Mpc}^{-1}, \Omega_{\mathrm{m}}=0.3$ and $\Omega_{\Lambda}=0.7$. Magnitudes are quoted in the AB system (Oke \& Gunn 1983).

\section{OBSERVATIONS}

\subsection{Data}

In selecting our small sample of bright, magnified $z \sim 7$ galaxies, we make use of the deep HST observations available over the first 23 clusters in the CLASH multicycle treasury program (GO 12101: PI Postman), A1689 and A1703 (GO 11802; PI: Ford), the Bullet cluster (GO 11099; PI: Bradac), and 9 clusters from the Kneib et al. (GO 11591) program. The CLASH cluster fields are each covered with 20 orbit HST observations spread over 16 bands using the Advanced Camera for Surveys (ACS; $B_{435}, g_{475}, V_{606}, r_{625}, i_{775}, I_{814}$, and $\left.z_{850}\right)$, Wide Field Camera WFC3/UVIS $\left(U V_{225}, U V_{275}, U_{336}\right.$ and $\left.U_{390}\right)$ and WFC3/IR instrument $\left(Y_{105}, J_{110}, J_{125}, J H_{140}\right.$ and $\left.H_{160}\right)$. A1703 was covered with 22 orbits of ACS and WFC3/IR $\left(B_{435}, g_{475}, V_{606}, r_{625}, i_{775}, z_{850}, J_{125}, H_{160}\right)$, A1689 was covered with 54 orbits $\left(B_{435}, r_{625}, i_{775}, I_{814}, z_{850}\right.$, $\left.J_{125}, J_{140}, H_{160}\right)$, the Bullet cluster was covered with 16 orbits $\left(V_{606}, i_{775}, z_{850}, J_{110}, H_{160}\right)$ and clusters in the Kneib et al. program were covered with 6 orbits $\left(I_{814}, J_{110}, H_{160}\right)$. HST mosaics were produced using the Mosaicdrizzle pipeline (see Koekemoer et al. 2011 for further details), and individual bands in the deep imaging data reach $5 \sigma$ depths of 26.4-27.7 mag (0.4-diameter aperture).

Deep Spitzer/IRAC observations of our fields in the [3.6] and [4.5] bands were provided by the ICLASH (GO 80168; Bouwens et al. 2011), the Ultra-Deep IRAC imaging of Massive Lensing Galaxy Clusters (GO 20439; PI: Egami) and the Spitzer IRAC Lensing Survey program (GO 60034; PI: Egami). The typical exposure time per cluster was $3.5-5 \mathrm{hr}$ per band, allowing us to reach $26.5 \mathrm{mag}$ at $1 \sigma$. Reductions of the IRAC observations used in this paper were performed with MOPEX (Makovoz \& Khan 2005).

\subsection{Photometry and Selection}

The photometry we obtain for sources in our cluster fields follows a similar procedure as described in Bouwens et al. (2012b). In short, we run the SExtractor software (Bertin \& Arnouts 1996) in dual-image mode. The detection images are 
constructed from all bands redward of the Lyman break (i.e., $Y_{105}, J_{110}, J_{125}, J H_{140}$ and $\left.H_{160}\right)$. After point-spread function (PSF)-matching the observations to the $H_{160}$-band PSF, colors are measured in Kron-like apertures and total magnitudes derived from 0 .' 6 diameter circular apertures.

Our initial source selection is based on the Lyman-break technique (Steidel et al. 1999), with the requirement that the source drops out in the $I_{814}$ band. Specifically, our requirements for $z \sim 6-7$ sources are

$$
\left(I_{814}-J_{110}>0.7\right) \wedge\left(J_{110}-J H_{140}<0.45\right) .
$$

For sources in the CLASH program, we require $H_{160}<26 \mathrm{AB}$, while we select sources to the brighter magnitude limit $H_{160}<$ $25 \mathrm{AB}$ in all other fields to ensure good photometric redshift constraints for all our sources. We also require sources to have either a nondetection in the $V_{606}$ band $(<2 \sigma)$ or to have a very strong Lyman break, i.e., $V_{606}-J_{125}>2.5$. We require sources to be undetected in the optical $\chi^{2}$ image (Bouwens et al. 2011) we construct from the observations blueward of the $r_{625}$ band. Finally, we require the SExtractor stellarity parameter (equal to 0 and 1 for extended and point sources, respectively) in the $J_{110}$ band be less than 0.92 to ensure that our selection is free of contamination by stars.

To identify those sources where we can obtain clean restframe optical stellar continuum, we also require that sources have a best-fit photometric redshift between $z=6.6$ and 7.0, as determined by the photometric redshift software EAZY (Brammer et al. 2008). All available HST photometry (i.e., 16 bands for CLASH clusters) is used in the redshift determinations. No use of the Spitzer/IRAC photometry is made in the photometric redshift determination to avoid coupling the selection of our sources to the [3.6]-[4.5] colors we will later measure. We use templates of young stellar populations with no Ly $\alpha$ emission.

Strong Ly $\alpha$ emission can systematically influence the photometric redshift estimate. However, we emphasize that any potential sources from outside our desired redshift interval that could be in our sample because of uncertainties in the photometric redshift estimate would serve only to increase the flux in the [4.5] band and redden the [3.6]-[4.5] color (i.e., because of contamination in the [4.5] band of $\mathrm{H} \alpha$ at $z<6.6$ and [O III] $+\mathrm{H} \beta$ at $z>7.0$ ). Correcting for this possible source of interlopers would result in higher EWs and sSFRs than in the case of no contamination. This reinforces the point we will make in Section 3 that the EWs we derive for the $[\mathrm{O} \mathrm{III}]+\mathrm{H} \beta$ emission and the sSFRs are strong lower limits on the actual values.

Figure 1 shows the redshift range where we would expect the strongest emission lines, $\mathrm{H} \alpha, \mathrm{H} \beta$, [O III] $\lambda \lambda 4959,5007$ and [O II] $\lambda 3727$, to impact the [3.6] and [4.5] fluxes. The top panel indicates which lines fall in specific IRAC filters at a given redshift, while the bottom panel indicates the estimated [3.6]-[4.5] color offset caused by the various emission lines. We select sources in the redshift range $z_{\text {phot }}=6.6-7.0$, where we know that both [O III] and $\mathrm{H} \beta$ fall in [3.6], while [4.5] falls exactly between [O III] and $\mathrm{H} \alpha$ where no significant emission lines are present (see for example Figure 2).

\subsection{IRAC Photometry}

Photometry of sources in the available Spitzer/IRAC data over our fields is challenging because of the blending with nearby sources from the broad PSF. We therefore use the automated cleaning procedure described in Labbé et al. (2010a,

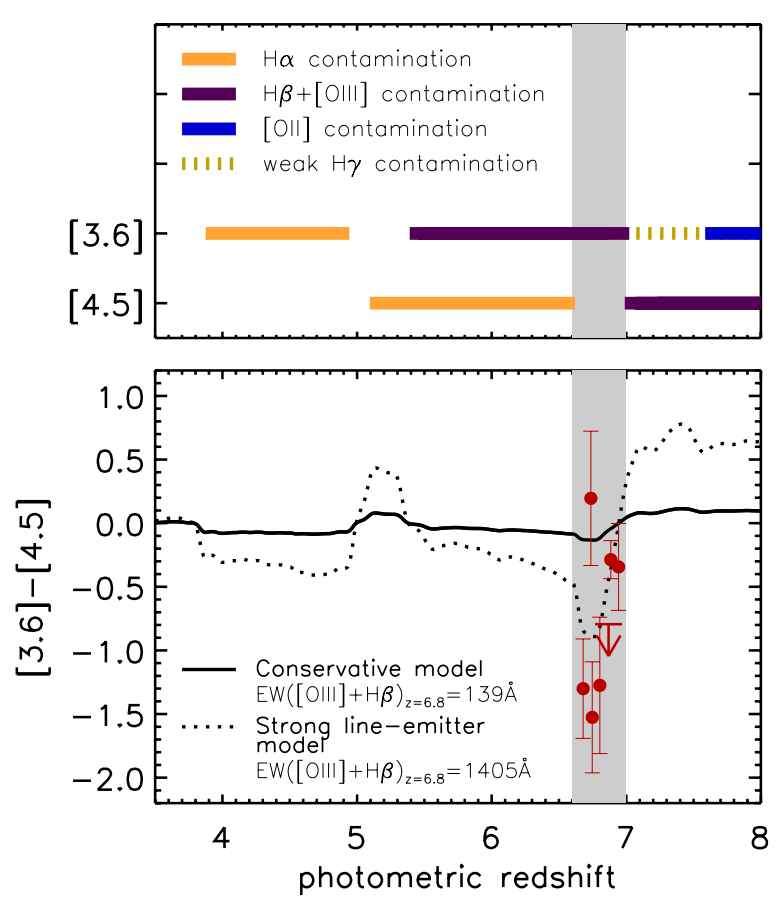

Figure 1. Impact of emission lines on the [3.6] and [4.5] band fluxes and our strategy for deriving sSFRs and $[\mathrm{O}$ III] $+\mathrm{H} \beta$ EWs from our $z \sim 7$ sample. Top panel: the redshift range over which strong nebular emission lines, $\mathrm{H} \alpha$, $\mathrm{H} \beta$, [O III], and [O II], will contaminate the [3.6] and [4.5] flux of galaxies. Bottom panel: the expected [3.6]-[4.5] colors as a function of redshift due to nebular emission lines. The solid and dotted lines show the expected color assuming relatively low EWs, i.e., $\mathrm{EW}_{0}([\mathrm{O} \mathrm{III}]+\mathrm{H} \beta) \sim 140 \AA$, and assuming strong evolution, i.e., $\mathrm{EW}_{0}\left(\left[\mathrm{O}_{\mathrm{III}}\right]+\mathrm{H} \beta\right) \propto(1+z)^{1.8} \AA$ (Fumagalli et al. 2012), respectively, similar to the models considered in González et al. (2014) and Stark et al. (2013). We select sources in the redshift range $z_{\text {phot }}=6.6-7.0$, where [O III] $\lambda \lambda 4959,5007$ and $\mathrm{H} \beta$ are present in [3.6], while [4.5] receives no significant contamination from nebular emission lines, falling exactly in between the $\mathrm{H} \alpha$ and [O III] lines. The solid red circles and $1 \sigma$ upper limit show the observed colors in our sample. We find that most sources show blue [3.6]-[4.5] colors, falling in the range between our two models. Four sources from our sample exhibit extremely blue rest-frame optical colors, with [3.6]-[4.5] $\lesssim-0.8$, indicating contamination of [O III] $+\mathrm{H} \beta$ with a mean EW of $>1582 \AA$ (see Section 3.2), even higher than using the Fumagalli et al. 2012 extrapolation indicated by the dotted line. Two sources at $z \sim 6.75$ have been offset by $\Delta z=0.05$ for clarity.

(A color version of this figure is available in the online journal.)

2010b). In short, we use the high-spatial-resolution HST images as a template with which to model the positions and flux profiles of the foreground sources. The flux profiles of individual sources are convolved to match the IRAC PSF and then simultaneously fit to all sources within a region of $\sim 13$ " around the source. Flux from all the foreground galaxies is subtracted and photometry is performed in $2^{\prime \prime} .5$ diameter circular apertures. We apply a factor of $\sim 2.0 \times$ correction to account for the flux outside of the aperture, based on the radial light profile of the PSF. Figure 3 shows the cleaned IRAC images of our sample. Our photometric procedure fails when contaminating sources are either too close or too bright. Sources with badly subtracted neighbors are excluded. In total, clean photometry is obtained for $78 \%$ of the sources, resulting in seven sources in our final selection (excluding only one source behind RXJ1347 and one source behind MACS1206 from our sample).

\section{RESULTS}

Our search for bright $\left(H_{160} \lesssim 26\right)$ LBGs in the redshift range $z \sim 6.6-7.0$ behind strong lensing clusters results in nine candidates. One of the sources in our $z \sim 7$ sample was 


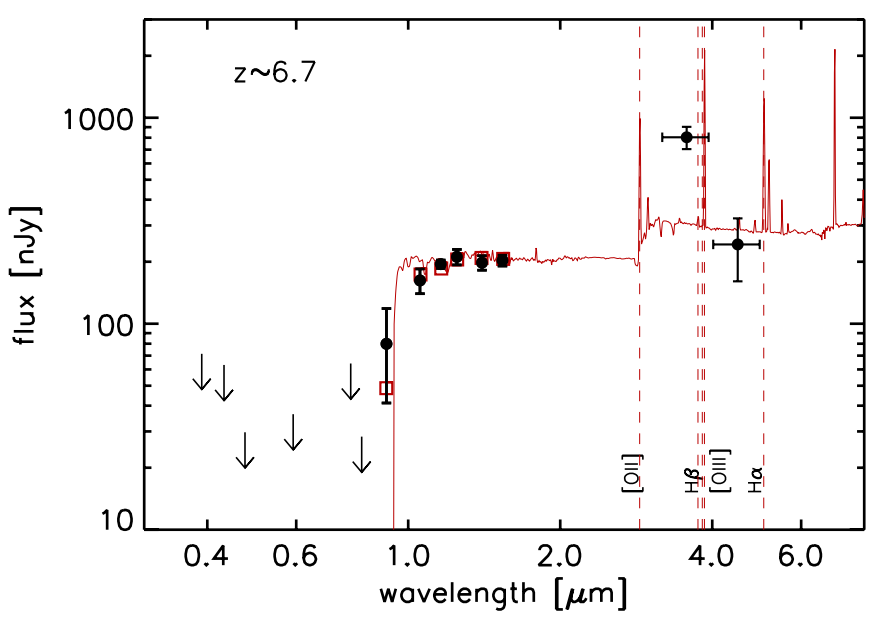

Figure 2. Observed HST + Spitzer fluxes (black circles) and $2 \sigma$ upper limits (black arrows) with the best-fit spectrum (red) from our photometric redshift estimate (Section 2.2) for one $z \sim 7$ candidate rxj1347Z-7362045151 that exhibits a very blue [3.6]-[4.5] color. Because of the brightness of sources in our sample and the many HST filters with deep observations in the CLASH program, their photometric redshifts are well determined. This is important for establishing that our selected sources are likely in the target redshift window. The [3.6] and [4.5] bands that are shown are not used for the photo- $z$ determination in order to avoid coupling the selection of our sources to the [3.6]-[4.5] colors. (A color version of this figure is available in the online journal.)

previously reported by Bradley et al. (2012a) based on a study of A1703. For seven sources, we obtain reasonably clean IRAC photometry, as shown in the postage stamps in Figure 3. The properties of the sources are summarized in Tables 1 and 2, and their $H_{160}$ band magnitude ranges from 24.3 to 25.7. Typical magnification factors, $\mu$, for our sources are $\sim 2-9$, using the lensing models of Zitrin et al. $(2010,2011)$ and A. Zitrin et al. (in preparation). Although the magnification of the sources improves the signal-to-noise ratio $(\mathrm{S} / \mathrm{N})$ of our measurements, we stress that measurements of emission line EWs and sSFRs depend only on the colors of the spectral energy distribution (SED) and therefore are not affected by uncertainties in the model magnification factors.

\section{1. [3.6]-[4.5] Color Distribution and Nebular Emission Lines}

Our selection of sources in the redshift range $z \sim 6.6-7.0$ provides us with the valuable opportunity to establish the typical EW of the nebular emission lines in $z \gtrsim 6$ sources through a comparison of the flux in [3.6] and [4.5]. For a given EW, we calculate the [3.6]-[4.5] color by assuming that the observed flux in one filter can be approximated by the following:

$$
\begin{aligned}
f_{v, \mathrm{obs}} & =f_{v, \text { continuum }} \cdot x_{\mathrm{EW}} \\
x_{\mathrm{EW}} & =\left(1+\sum_{\text {lines }, i} \frac{E W_{0, i} \cdot(1+z) \cdot R\left(\lambda_{\mathrm{obs}, i}\right)}{\lambda_{\mathrm{obs}, \mathrm{i}} \int R(\lambda) / \lambda d \lambda}\right) .
\end{aligned}
$$

Here, $f_{v, \text { continuum }}$ is the intrinsic flux of the stellar continuum, while $f_{v, \text { obs }}$ is the observed flux of the filter. Furthermore, $\lambda_{\text {obs, } i}$ is the observed wavelength of the line, and $R(\lambda)$ is the response curve of the filter. We include all nebular emission lines listed in Anders \& Fritze-v. Alvensleben (2003) and the hydrogen Balmer lines. We assume fixed flux ratios between the emission lines based on the tabulated values in Anders \& Fritze-v. Alvensleben (2003) for $0.2 Z_{\odot}$ metallicity and assuming case $\mathrm{B}$ recombination. The observed [3.6]-[4.5] color then

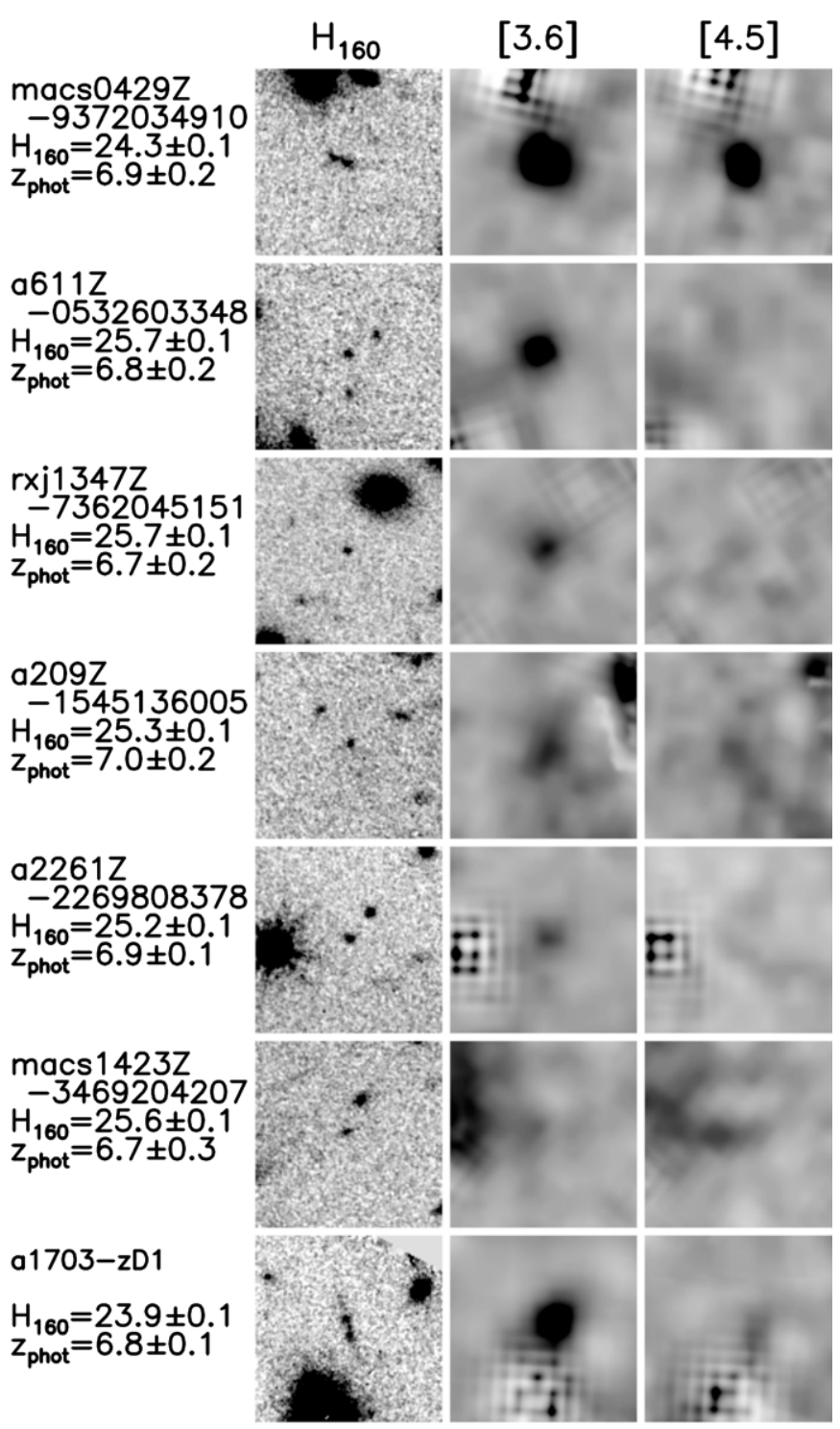

Figure 3. $H S T H_{160}$, Spitzer/IRAC [3.6], and [4.5] postage stamp images (6.'5 $\times$ 6 .'5) of our sample of bright, magnified $z \sim 6.6-7.0$ galaxies behind clusters The IRAC postage stamps have already been cleaned for contamination from neighboring sources (Section 2.3). It is obvious that a large fraction of the sources in our selection are much brighter at $3.6 \mu \mathrm{m}$ than at $4.5 \mu \mathrm{m}$.

simplifies to the following:

$$
[3.6]-[4.5]=([3.6]-[4.5])_{\text {continuum }}-2.5 \cdot \log \left(\frac{x_{\mathrm{EW}, 3.6}}{x_{\mathrm{EW}, 4.5}}\right) \text {. }
$$

LBGs at high redshift are expected to exhibit a flat optical stellar continuum based on stellar population synthesis models. In these models, young galaxies with typical ages between 50 and $200 \mathrm{Myr}$ and low dust extinction, e.g., $E(B-V) \sim 0.1$, will have a $([3.6]-[4.5])_{\text {continuum }}$ color of $\sim 0 \pm 0.1 \mathrm{mag}$. However, extremely young (i.e., $\sim 3 \times 10^{6} \mathrm{yr}$ ), dust-free galaxies can

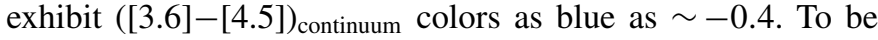
conservative, we adopt this for the color of the underlying stellar continuum and assume that any bluer [3.6]-[4.5] color arises from the impact of emission lines to establish robust lower limits.

In the bottom panel of Figure 1, the dotted line shows a prediction of the observed optical color due to emission lines, 
Table 1

$z \sim 6.6-7.0$ Candidates Included in This Work

\begin{tabular}{|c|c|c|c|c|c|}
\hline $\mathrm{ID}^{\mathrm{a}}$ & R.A. & Decl. & $z_{\text {phot }}$ & $\mu^{\mathrm{b}}$ & $M_{\mathrm{UV}}^{\mathrm{c}}$ \\
\hline macs0429Z-9372034910 & $04: 29: 37.20$ & $-2: 53: 49.10$ & $6.9 \pm 0.2$ & $2.5 \pm 0.2$ & $-21.6 \pm 0.1$ \\
\hline a611Z-0532603348 & 08:00:53.26 & $36: 03: 34.8$ & $6.7 \pm 0.2$ & $1.8 \pm 0.1$ & $-20.5 \pm 0.1$ \\
\hline rxj1347Z-7362045151 & $13: 47: 36.20$ & $-11: 45: 15.1$ & $6.7 \pm 0.2$ & $2.7 \pm 0.2$ & $-20.1 \pm 0.1$ \\
\hline a209Z-1545136005 & $01: 31: 54.51$ & $-13: 36: 00.5$ & $6.9 \pm 0.2$ & $1.2 \pm 0.0$ & $-21.4 \pm 0.1$ \\
\hline a2261Z-2269808378 & $17: 22: 26.99$ & $32: 08: 37.8$ & $6.9 \pm 0.1$ & $5.6 \pm 1.7$ & $-19.9 \pm 0.3$ \\
\hline macs1423Z-3469204207 & $14: 23: 46.92$ & 24:04:20.7 & $6.7 \pm 0.3$ & $4.7 \pm 1.0$ & $-19.6 \pm 0.3$ \\
\hline
\end{tabular}

Notes.

a Two sources from our initial Lyman-break selection displayed bad residuals in the IRAC data. The two rejected sources are RXJ1347I-7332751567 (R.A. 13:47:33.27, decl. -11:45:15.67) and MACS1206Z-6100148441 (R.A. 12:06:10.01, decl. -8:48:44.1).

b The lens models for RXJ1347, MACS0429, A611, and A209 are made with an improved version of the method described in Zitrin et al. (2009) and will be published in A. Zitrin et al. (in preparation). The model for A2261 is described Coe et al. (2012). The model for MACS1423 is described in Zitrin et al. (2011), but here, we use a refined model (The CLASH collaboration, in preparation). The model for A1703 is described in Zitrin et al. (2010). Errors on the magnification factors are typical errors at a given $\mu$, calculated using the modeled uncertainties of A383, A611, MS2137, and MACS1423 (A. Zitrin et al., in preparation).

${ }^{c}$ The intrinsic UV magnitude is derived from the $H$-band magnitude, corrected for the magnification, $\mu$. The quoted uncertainty includes the estimated uncertainty in $\mu$.

d Although this source is very compact, our size measurements indicate that it is slightly extended.

e Previously reported in Bradley et al. (2012a).

Table 2

Properties of the Mean Stack and the Individual Sources in Our Sample of $z \sim 6.6-7.0$ Galaxies

\begin{tabular}{|c|c|c|c|c|c|c|}
\hline ID & $H_{160}$ & $H_{160}-[4.5]$ & {$[3.6]-[4.5]$} & $\beta^{\mathrm{a}}$ & $\mathrm{EW}([\mathrm{O} \mathrm{III}]+\mathrm{H} \beta)[\AA]^{\mathrm{b}}$ & $\log \left(\mathrm{sSFR} / \mathrm{Gyr}^{-1}\right)$ \\
\hline macs0429Z-9372034910 & $24.3 \pm 0.1$ & $0.7 \pm 0.1$ & $-0.3 \pm 0.1$ & $-1.4 \pm 0.4$ & $\cdots$ & $-7.8_{-0.7}^{+0.8}$ \\
\hline a611Z-0532603348 & $25.7 \pm 0.1$ & $0.3 \pm 0.4$ & $-1.5 \pm 0.4$ & $-1.5 \pm 0.5$ & $>1905$ & $>-8.1^{\mathrm{c}}$ \\
\hline rxj1347Z-7362045151 & $25.7 \pm 0.1$ & $0.2 \pm 0.4$ & $-1.3 \pm 0.4$ & $-2.2 \pm 0.5$ & $>1363$ & $-7.6_{-1.0}^{+0.6}$ \\
\hline a209Z-1545136005 & $25.3 \pm 0.1$ & $1.0 \pm 0.3$ & $-0.3 \pm 0.3$ & $-2.7 \pm 0.6$ & $\ldots$ & $<-8.1^{\mathrm{d}}$ \\
\hline a2261Z-2269808378 & $25.2 \pm 0.1$ & $<0.0$ & $<-0.8$ & $-2.0 \pm 0.3$ & $>1043$ & $>-7.8^{c}$ \\
\hline macs1423Z-3469204207 & $25.6 \pm 0.1$ & $1.0 \pm 0.3$ & $0.2 \pm 0.5$ & $-1.2 \pm 0.8$ & $\ldots$ & $<-7.0^{\mathrm{d}}$ \\
\hline a1703-zD1 & $23.9 \pm 0.1$ & $0.2 \pm 0.2$ & $-1.3 \pm 0.5$ & $-1.4 \pm 0.3$ & $>1304$ & $-6.4_{-0.1}^{+0.4}$ \\
\hline Mean stack & $25.5 \pm 0.1$ & $0.2 \pm 0.2$ & $-0.9 \pm 0.3$ & $-1.9 \pm 0.3$ & $>637$ & $-7.3_{-0.7}^{+0.3}$ \\
\hline
\end{tabular}

Notes. Upper and lower limits are $1 \sigma$.

a The UV-continuum slope $\beta$ is estimated using a log-linear fit to the $J_{125}, J H_{140}$ and $H_{160}$ fluxes.

${ }^{\mathrm{b}}$ Estimates are lower limits assuming the bluest possible continuum color of [3.6]-[4.5] $=-0.4$, see Section 3.1.

${ }^{c}$ We can put a lower limit only on the sSFR of this source because of the blue $H_{160}-$ [4.5] color in combination with the large error bar on the [4.5]

flux, i.e., the fit of the stellar population is consistent with an arbitrarily young age.

${ }^{\mathrm{d}}$ We can put an upper limit only on the sSFR of this source with our data. The lower limit is set by the age of the universe $\left(\sim 1.3 \mathrm{Gyr}{ }^{-1}\right.$ at $\left.z \sim 6.8\right)$.

using Equations (1) and (2) and assuming a flat continuum, for a model of strongly increasing rest-frame emission line EWs as a function of redshift (dotted line), with $\mathrm{EW}_{0}([\mathrm{O} \mathrm{III}]+\mathrm{H} \beta) \propto$ $(1+z)^{1.8} \AA$, based on the evolution in $\mathrm{EW}_{0}(\mathrm{H} \alpha)$ found by Fumagalli et al. (2012) for star-forming galaxies over the redshift range $0 \lesssim z \lesssim 2$. The red points in Figure 1 show the observed colors for our sample. Most of our sources show quite blue [3.6]-[4.5] colors, and essentially all of them are bluer than expected based on a conservative model of constant rest-frame $\mathrm{EW}$ (solid black line: i.e., assuming no evolution from $z \sim 2$ where $\mathrm{EW}_{0}([\mathrm{O} \mathrm{III}]+\mathrm{H} \beta) \sim 140 \AA$, derived from the $\mathrm{H} \alpha$ EWs found by Erb et al. 2006). It is interesting that three of the sources from our sample have [3.6]-[4.5] colors even bluer than expected at $z \sim 6.7-6.8$ for the model from Fumagalli et al. (2012) with $\mathrm{EW}_{0}(\mathrm{H} \alpha) \propto(1+z)^{1.8} \AA$. Four of the sources have [3.6]-[4.5] colors bluer than -0.8 . Because we would expect galaxies to show such extreme [3.6]-[4.5] colors only in the narrow redshift range $z \sim 6.6-7.0$, this assures us that our selection can effectively identify sources in the desired redshift range.

\subsection{Inferred $[O \mathrm{III}]+H \beta E W s$ of $z \sim 7$ Galaxies From the Mean SED}

To obtain our best measurement of the [4.5] flux and hence stellar continuum light from $z \sim 7$ galaxies, we construct a mean SED that can be directly compared with studies of the mean restframe optical properties of $z \gtrsim 4$ galaxies in the literature (e.g., González et al. 2012, 2014; Stark et al. 2013). We use a mean stack of the clean [3.6] and [4.5] images after dividing by the observed rest-frame UV luminosity (the geometric mean of the $J_{125}, J H_{140}$ and $H_{160}$ luminosities). We measure the flux in a $2^{\prime \prime} .5$ diameter aperture on the stacked image and apply an aperture correction measured from the PSF images $(\sim 2.0 \times)$. Also, we use the mean of the individual measurements of the HST bands, after dividing by the rest-frame UV-luminosity. The mean SED of our stacked $z \sim 6.8$ sample is shown in Figure 4. Errors are obtained through bootstrap resampling.

We use the stacked detections in the IRAC bands to evaluate the mean contribution of the emission lines. From the mean [3.6]-[4.5] color, we estimate the $[\mathrm{O}$ III] $+\mathrm{H} \beta$ EW by assuming 


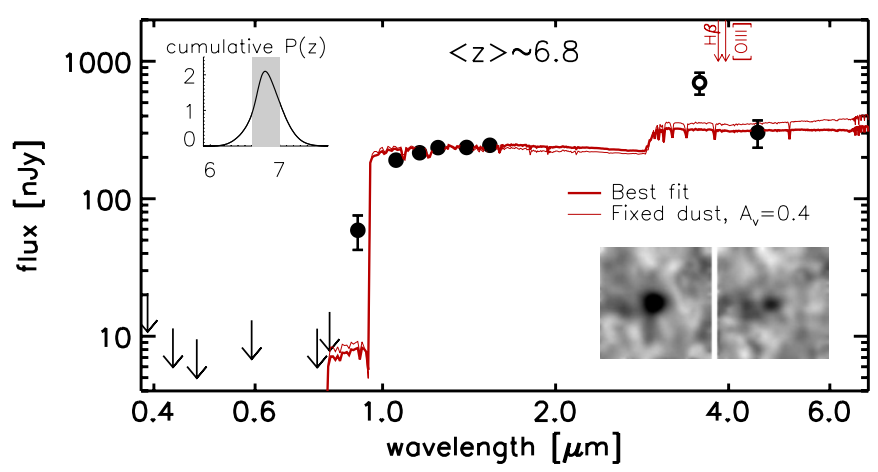

Figure 4. Mean-stacked SED of our $z \sim 6.6-7.0$ sample. The inset images on the bottom right show the stacked photometry of the [3.6] (left) and [4.5] (right) bands. The mean [3.6]-[4.5] color for our sample is $\sim-0.9$ and indicates significant line contamination for the typical $z \sim 7$ galaxy. The top left inset panel indicates the sum of the redshift probability distributions for the sources in our sample. A small fraction of the sources in our photometric redshift selection are expected to lie outside the target range $z \sim 6.6-7.0$ (because of redshift uncertainties). Any such contamination would make the [3.6]-[4.5] colors redder because of the contribution of $\mathrm{H} \alpha$ (at $z<6.6$ ) or $[\mathrm{O} \mathrm{III}]+\mathrm{H} \beta$ (at $z>7$ ) emission to the [4.5] flux. The thick, red line indicates the best fit SED to our observed photometry, excluding the [3.6] flux from the fit. The thin, red line indicates a best fit SED when the dust content is fixed to $A_{V}=0.38$, similar to the assumptions used in Bouwens et al. (2012b), Stark et al. (2013), González et al. (2014) and Labbé et al. (2013).

(A color version of this figure is available in the online journal.)

that our entire sample is at $z=6.76$, where we expect the most extreme colors because [4.5] is completely free of emission lines, whereas [3.6] is contaminated by both the [O III] doublet and $\mathrm{H} \beta$. In practice, this results in an underestimate of the intrinsic line strength because we know that the [O III] lines start to drop out of [3.6] at $z \sim 6.9-7.0$. Therefore, we expect a less extreme [3.6]-[4.5] color for a given mean EW at $z \sim 6.9-7.0$ than at $z \sim 6.7-6.8$. It is also possible that because of uncertainties in the photometric redshifts, sources outside of our target redshift range have been included in our selection; therefore, the measurement of the [4.5] flux is contaminated by either $\mathrm{H} \alpha(z<6.6)$ or [O III] $(z>7)$. This would also make the mean [3.6]-[4.5] color redder and accordingly make the emission lines appear to be less extreme.

The mean observed [3.6]-[4.5] color for our sample is $-0.9 \pm 0.3$ (error obtained through bootstrap resampling). In the most conservative estimate, we assume that the underlying stellar continuum exhibits a [3.6]-[4.5] color of $\sim-0.4$; therefore, the [O III] and $\mathrm{H} \beta$ are responsible for a color of [3.6]-[4.5] $\sim-0.5$, which would give a robust lower limit of $\mathrm{EW}_{0}([\mathrm{O} \mathrm{III}]+\mathrm{H} \beta) \gtrsim 637 \AA$ for the mean $z \sim 7$ galaxy distribution. The $[\mathrm{O} \mathrm{III}]+\mathrm{H} \beta \mathrm{EW}$ we estimate here is equivalent to $\mathrm{EW}_{0}\left(\mathrm{H} \alpha+\left[\mathrm{N}_{\mathrm{II}}\right]\right) \gtrsim 495 \AA$, adopting the tabulated values from Anders \& Fritze-v. Alvensleben (2003) for $0.2 Z_{\odot}$ metallicity and assuming case $\mathrm{B}$ recombination.

The mean observed [3.6]-[4.5] color for our four bluest sources is $-1.4 \pm 0.4$. If we assume again a very blue underlying continuum (i.e., -0.4), line emission would be responsible for a color of [3.6]-[4.5] $\sim-1.0$, consistent with a robust lower limit of $\mathrm{EW}_{0}([\mathrm{O} \mathrm{III}]+\mathrm{H} \beta) \gtrsim 1582 \AA$ for these sources. The individual lower limits for these blue sources are listed in Table 2. Although the four bluest sources in our sample are, given their extreme colors, almost certainly at a redshift $z \sim 6.7-6.8$, it is unclear whether the three other sources are less extreme because of a lower [O III $]+\mathrm{H} \beta \mathrm{EW}$ or simply because they lie in a different redshift range (i.e., $z \gtrsim 6.9$ or close to $z \sim 6.6$ ), where for a given EW we expect somewhat redder colors.

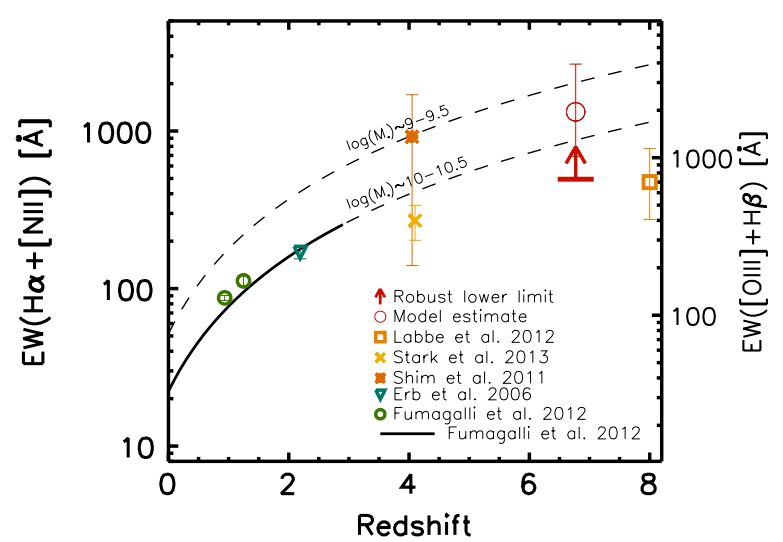

Figure 5. Constraint on the evolution of $\left[\mathrm{O}_{\mathrm{III}}\right]+\mathrm{H} \beta$ EWs (and equivalent $\mathrm{H} \alpha$ EWs) from our stacking analysis and references from the literature (Erb et al. 2006; Shim et al. 2011; Fumagalli et al. 2012; Stark et al. 2013; Labbé et al. 2013). The robust lower limit (red arrow) assumes all sources are at $z=6.76$ where the [3.6]-[4.5] color is expected to be the most extreme for a given set of EWs and that the underlying stellar continuum has a [3.6]-[4.5] color of $\sim-0.4$ (which would be the case only if all galaxies have an age of $\sim 3 \times 10^{6} \mathrm{yr}$ ). Any bluer [3.6]-[4.5] color would therefore arise from the impact of the $[\mathrm{O} I I I]+\mathrm{H} \beta$ emission lines on the [3.6] flux. For the model estimate (open, red circle), we model the effects of a broader redshift distribution as described in Section 3.2. For comparison with lower redshift estimates, we convert our EWs to EW(H $\alpha+[\mathrm{N}$ II $])$ using the conversion factors from Anders \& Fritze-v. Alvensleben (2003). The high EW inferred from our mean stacked sample indicates significantly stronger emission lines than observed at redshift $z \sim 0-2$, possibly consistent with an extrapolation of the trends with redshift and mass found by Fumagalli et al. (2012, indicated by the dashed, black lines). (A color version of this figure is available in the online journal.)

To obtain a good estimate of the EW, we need detailed knowledge of the redshift distribution and the underlying stellar continuum color. Given our lack of deep spectroscopy for our sample, we make some reasonable assumptions to obtain a model estimate of the rest-frame EW of our sample. We assume that the redshift probability distribution of our sources is given by the sum of the probability distributions obtained from our photometric redshift code, corrected for the fact that galaxies are more difficult to observe at higher redshift scaling roughly as $(d \log \phi / d z)=0.3$ (Bouwens et al. 2012a) and assuming no sources are outside our desired redshift range $z \sim 6.6-7.0$. We use this redshift probability distribution in our sample to estimate the expected [3.6]-[4.5] color distribution, for a given mean $\mathrm{EW}_{0}([\mathrm{O} \mathrm{III}]+\mathrm{H} \beta)$ and 0.3 dex scatter around the mean. We assume the underlying continuum color is -0.25 , as would be expected for a galaxy age of $\sim 10 \mathrm{Myr}$. We randomly draw sources from the distribution and calculate the $68 \%$ likelihood of finding a mean $\langle[3.6]-[4.5]\rangle$ color given a total of seven observed sources and the observed photometric errors. On the basis of this modeling, the observed [3.6]-[4.5] -0.9 mag color is consistent with a possible $\mathrm{EW}_{0}([\mathrm{O} \mathrm{III}]+\mathrm{H} \beta)$ EW of $\sim 1806_{-863}^{+1826} \AA$. This is equivalent to $\mathrm{EW}_{0}(\mathrm{H} \alpha+[\mathrm{N} \mathrm{II}]) \sim$ $1323_{-632}^{+1338} \AA$, adopting the same conversion factor from Anders \& Fritze-v. Alvensleben (2003) assumed earlier.

The aforementioned modeling we perform indicates that the true $\mathrm{EW}_{0}([\mathrm{O} \mathrm{III}]+\mathrm{H} \beta)$ may be $\sim 2-3 \times$ larger than our robust lower limit of $637 \AA$. Figure 5 compares our results with other determinations from the literature (Erb et al. 2006; Shim et al. 2011; Fumagalli et al. 2012; Stark et al. 2013; Labbé et al. 2013). The solid and dashed lines in Figure 5 show the expected evolution of the $\mathrm{H} \alpha+[\mathrm{N}$ II] EWs extrapolating the evolution found in Fumagalli et al. (2012) at $z \sim 0-2$. We note, however, that a direct comparison is difficult to make because the Fumagalli et al. (2012) relation was derived 
for galaxies in the mass range $M_{*}=10^{10}-10^{10.5} M_{\odot}$, while we are probing galaxies in the mass range $10^{9}-10^{9.5} M_{\odot}$. As a reference, we show the possible evolution of galaxies $M_{*}=10^{9}-10^{9.5} M_{\odot}$ (top dashed line), using the same scaling with redshift $\mathrm{EW}_{0}(\mathrm{H} \alpha+[\mathrm{N}$ II $]) \propto(1+z)^{1.8} \AA$ but extrapolating the normalization to lower masses, based on the mass trend in the SDSS-DR7 data derived in Fumagalli et al. (2012).

In general, the EWs we infer are in good agreement with extrapolations from previous results at lower redshift. However, our results are based on a UV-selected sample, which could yield different results from a mass-complete sample. It is clear nonetheless that our EWs estimates strongly support the high EWs used by Stark et al. (2013) and González et al. (2014) in correcting the SEDs of $z \sim 5-7$ samples to derive higher values of the sSFRs.

\subsection{Specific Star-formation Rates}

The redshift range where we select galaxies is the only redshift window at $z \gtrsim 5$ where we can probe the rest-frame stellar continuum light in an uncontaminated fashion, using the [4.5] IRAC band (see Figure 1). This allows us to estimate the sSFR with minimal contamination from emission lines.

Because of the low $\mathrm{S} / \mathrm{N}$ of the uncontaminated [4.5] band, we can set only weak constraints on the sSFRs of the individual galaxies (see Table 2). To optimize the $\mathrm{S} / \mathrm{N}$, we perform stellar population modeling of our stacked photometry, leaving out the [3.6] measurement. We performed the modeling with FAST (Kriek et al. 2009), using the Bruzual \& Charlot (2003, hereafter BC03) stellar populations synthesis models. We use a Salpeter (1955) IMF with limits $0.1-100 M_{\odot}$ and a Calzetti et al. (2000) dust law. We consider ages between $10 \mathrm{Myr}$ and the age of the universe at $z \sim 6.8$ and dust extinction between $A_{V}=0-2$. We assume a constant star-formation history and subsolar metallicity $\left(0.2 Z_{\odot}\right)$. For the mean stack, we fix the redshift to the median of the photometric redshifts, at $z=6.77$.

Given this freedom of parameters, the mean SED is best described by a fairly young galaxy (age $\lesssim 100 \mathrm{Myr}$ ) and reasonable dust $\left(A_{V} \sim 0.7\right)$ to fit both the small Balmer break $\left(H_{160}-[4.5] \sim 0.2\right)$ and moderately red UV-continuum slope $(\beta \sim-1.9)$, resulting in a notably high sSFR of $52_{-41}^{+50} \mathrm{Gyr}^{-1}$ (see the SED in Figure 4). However, the interpretation of this result is not straightforward. First, we have only the [4.5] band in the rest-frame optical to break the age-dust degeneracy. Given our modest sample, a range of models can still fit the data well. We obtain a possibly more insightful answer when we fix the dust to the expected value derived from the typical spread of UV-continuum slopes and the Meurer et al. (1999) law (e.g., Bouwens et al. 2012b) similar to the assumptions made in Bouwens et al. (2012b), Stark et al. (2013), González et al. (2014), and Labbé et al. (2013). This results in a dust content of $A_{V}=0.38 \pm 0.16$ using the latest numbers from Bouwens et al. (2013). In turn, we fix the dust content to $A_{V}=[0.22,0.38,0.54]$. The fit for $A_{V}=0.38$ is shown with the thin red line in Figure 4. We obtain a sSFR of $7_{-3}^{+7} \mathrm{Gyr}^{-1}$. Given that the reduced $\chi^{2}$ with the lowest dust content $A_{V}=0.22$ is only $\sim 1.3$ times higher than for our best fit $\left(\chi^{2}=1.79\right.$ versus $\left.\chi^{2}=1.33\right)$, we assume a conservative lower limit of the sSFR of $\gtrsim 4 \mathrm{Gyr}^{-1}$.

Alternatively, we can estimate the sSFR of our $z \sim 7$ sample from the $[\mathrm{O} \mathrm{III}]+\mathrm{H} \beta$ EWs we infer, by converting to $\mathrm{H} \alpha \mathrm{EW}$ assuming same line ratios from Anders \& Fritze-v. Alvensleben (2003) as described in Section 3.2. We use the Kennicutt (1998) relation to convert $\mathrm{H} \alpha$ luminosity to SFR, and we use BC03

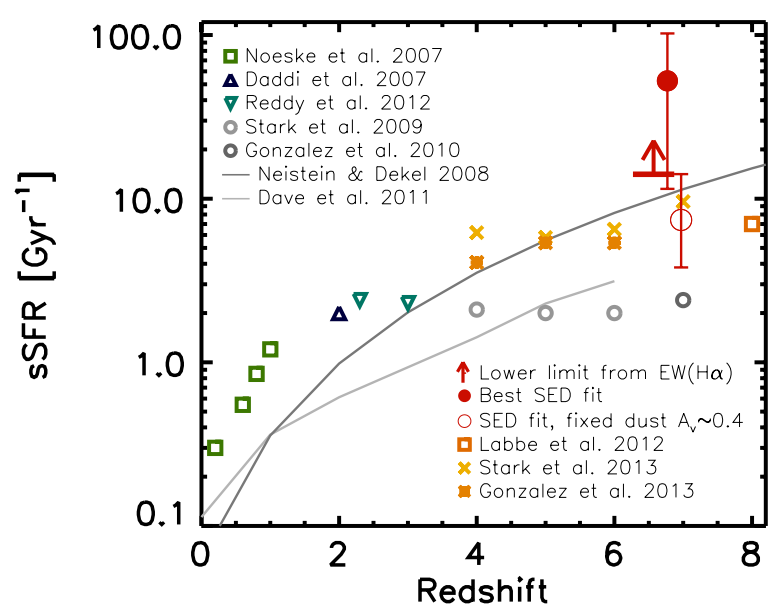

Figure 6. Evolution of the sSFR as a function of redshift, based on the fitted SED to our stacked photometry, excluding the [3.6] flux from the fit. Error bars are the $68 \%$ confidence interval, based on the photometric uncertainties. The filled red circle indicates the best fit using the assumptions described in Section 3.3, leaving dust as a free parameter. The open red circle indicates a fit where the dust content is fixed at $A_{V}=0.38 \pm 0.16$. We also show a lower limit (red arrow) on the sSFR we derive from the EW of [O III] $+\mathrm{H} \beta$, by converting to $\mathrm{H} \alpha$ assuming the line ratios from Anders \& Fritze-v. Alvensleben (2003). For context, we also include many previous sSFR results from the literature (Noeske et al. 2007; Daddi et al. 2007; Stark et al. 2009, 2013; González et al. 2010, 2014; Reddy et al. 2012; Labbé et al. 2013). Our results indicate possible strong evolution in the sSFRs from redshift $z \sim 2$ to $z \sim 7$, consistent with other recent results that are based on an extrapolation of the $z \sim 4 \mathrm{H} \alpha \mathrm{EW}$ distribution (Stark et al. 2013; González et al. 2014) or for single $z \sim 7$ galaxies (e.g., Ono et al. 2012; Tilvi et al. 2013; Ouchi et al. 2013). Our results are also in agreement with theoretical predictions (e.g., Neistein \& Dekel 2008 (dark gray line) and Davé et al. 2011 (light gray line)).

(A color version of this figure is available in the online journal.)

models (assuming no dust) to convert the rest-frame optical continuum light to stellar mass. Using these assumptions, we obtain a lower limit on the sSFR for our seven source samples and the bluest four sources of $\sim 14 \mathrm{Gyr}^{-1}$ and $\sim 130 \mathrm{Gyr}^{-1}$, respectively, on the basis of the robust lower limits on the $[\mathrm{O}$ III $]+\mathrm{H} \beta$ EW derived in Section 3.2.

Comparing with direct constraints at $z \sim 2$, we estimate $\gtrsim 2 \times$ evolution in the sSFR over this redshift range (see Figure 6), in good agreement with estimates at $z \sim 7$ on the basis of a few spectroscopically confirmed sources (Ono et al. 2012; Tilvi et al. 2013) and extrapolating the $\mathrm{H} \alpha$ EWs from lower redshifts (Yabe et al. 2009; Stark et al. 2013; González et al. 2014). Our derived constraint is also in agreement with theoretical models that predict the sSFR to follow the specific infall rate of baryonic matter (e.g., Neistein \& Dekel 2008).

\section{SUMMARY AND DISCUSSION}

In this paper, we present the strongest direct evidence yet for very high $[\mathrm{O} \mathrm{III}]+\mathrm{H} \beta$ EWs in the $z \sim 7$ galaxy population. We also simultaneously explore a strategy for obtaining a clean measurement of the sSFR at $z \sim 7$ on the basis of the stellar continuum flux measured in the [4.5] $\mu \mathrm{m}$ band-which is largely free of contamination from the strongest nebular lines. Nebular emission lines ([O $\mathrm{III}], \mathrm{H} \alpha, \mathrm{H} \beta$ ) and the extreme faintness of $z \gtrsim 5.5$ galaxies make it extremely challenging to establish the stellar masses and sSFRs of the typical galaxy at high redshift.

To overcome these issues, we have isolated a small sample of nine bright $\left(H_{160}<26 \mathrm{mag}\right)$, magnified galaxies in the redshift range $z \sim 6.6-7.0$ from CLASH and other programs, for which 
seven we can perform high-quality IRAC photometry. Galaxies with photometric redshifts in the range $z \sim 6.6-7.0$ are useful given that there, the [4.5] band from Spitzer/IRAC provides us with a clean measurement of the stellar continuum flux from galaxies in the rest-frame optical, free of contamination from dominant nebular emission lines (Figures 1 and 2).

For the mean source in our sample, we find that we can set a robust lower limit on the rest-frame $\mathrm{EW}$ of $[\mathrm{O} \mathrm{III}]+\mathrm{H} \beta$ of $637 \AA$. For this lower limit, we adopt the bluest conceivable [3.6]-[4.5] colors for the stellar continuum and assume that all sources in our sample are at $z=6.76$, where a given $[\mathrm{O} \mathrm{III}]+\mathrm{H} \beta$ EW would produce the most extreme [3.6]-[4.5] color. Use of a more realistic redshift distribution for our sample, i.e., consistent with the photometric redshift estimates and not assuming that all sources are at $z=6.76$, suggests that these lower limits may underestimate the true EWs by a factors of $\sim 2 \times$.

The four bluest sources in our selection (58\% of our sample) show evidence for even more extreme line emission, with [3.6]-[4.5] $\lesssim-0.8$. For these four sources, we can set a robust lower limit of $1582 \AA$ on the rest-frame EW in [O III] $+\mathrm{H} \beta$.

Extreme line emission with EWs greater than $1000 \AA$ has been found at lower redshift in low-mass galaxies (van der Wel et al. 2011; Atek et al. 2011). Our results are consistent with the idea that extreme line emission may be present in the typical star-forming galaxy at $z \sim 7$.

Furthermore, our [4.5] stack results imply a firm lower limit on the sSFR of $\sim 4 \mathrm{Gyr}^{-1}$ for star-forming galaxies at $z \sim 7$. If any sources from our $z \sim 6.6-7.0$ photometric redshift sample lie at lower or higher redshifts than this, it would imply even lower [4.5] $\mu \mathrm{m}$ fluxes for the stack and hence higher sSFRs. Compared with sSFRs measurements at $z \sim 2$ (Daddi et al. 2007; Reddy et al. 2012), this implies at least a $\gtrsim 2 \times$ evolution in the sSFR over the redshift range $z \sim 2$ to $z \sim 7$. Similar to a few other spectroscopically confirmed $z \sim 7$ galaxies in the literature (Ono et al. 2012; Tilvi et al. 2013), this provides strong evidence that the sSFRs at $z \sim 7$ are high.

We expect improvement in these results through the measurement of spectroscopic redshifts for our sample from deep spectroscopy. This should allow us to obtain an even cleaner selection of $z \sim 6.6-7.0$ galaxies from which to quantify the emission line contamination and sSFRs. Follow-up observations of our sample are facilitated by the fact that these candidates are typically $\sim 1$ mag brighter than similar candidates found in the field, making these efforts feasible in terms of the telescope time required.

Moreover, our bright $z \sim 7$ sample is small and the $\mathrm{S} / \mathrm{N}$ we have per source is still modest. Increases in sample size can come from shallow surveys over a larger numbers of clusters, such as those available from recent snapshot programs. S/N increases will come from very deep $H S T+$ Spitzer observations being taken by the Frontier Fields program and the SURF'S Up program (Bradac et al. 2012).

We thank Jeff Cooke, Rob Crain, Eichii Egami, Andrea Ferrara, Marijn Franx, Max Pettini, Norbert Pirzkal, and Vivienne Wild for interesting conversations. Eichii Egami independently discovered the same extreme [3.6]-[4.5] colors in at least one of the sources from the present sample. We thank Pascal Oesch for useful feedback on our manuscript. We acknowledge support from ERC grant HIGHZ No. 227749, an NWO Vrij Competitie grant, and the NASA grant for the CLASH MCT program. Support for A.Z. is provided by NASA through Hubble Fellowship grant no. HST-HF-51334.01-A awarded by STScI, which is operated by the Association of Universities for
Research in Astronomy, Inc., for NASA, under contract NAS 5-26555. Part of this work was also supported by contract research "Internationale Spitzenforschung II/2-6" of the Baden Württemberg Stiftung. The work of L.A.M. was carried out at Jet Propulsion Laboratory, California Institute of Technology, under a contract with NASA.

\section{REFERENCES}

Anders, P., \& Fritze-v. Alvensleben, U. 2003, A\&A, 401, 1063

Atek, H., Siana, B., Scarlata, C., et al. 2011, ApJ, 743, 121

Bertin, E., \& Arnouts, S. 1996, A\&AS, 117, 393

Bouwens, R., Bradley, L., Zitrin, A., et al. 2012a, ApJ, submitted (arXiv:1211.2230)

Bouwens, R., Zheng, W., Moustakas, L., et al. 2011, Sptz Prop, 80168

Bouwens, R. J., Illingworth, G. D., Franx, M., \& Ford, H. 2007, ApJ, 670, 928

Bouwens, R. J., Illingworth, G. D., Oesch, P. A., et al. 2011, ApJ, 737, 90

Bouwens, R. J., Illingworth, G. D., Oesch, P. A., et al. 2012b, ApJ, 754, 83

Bouwens, R. J., Illingworth, G. D., Oesch, P. A., et al. 2013, ApJ, submitted (arXiv:1306.2950)

Bowler, R. A. A., Dunlop, J. S., McLure, R. J., et al. 2012, MNRAS, 426, 2772

Bradac, M., Gonzalez, A., Schrabback, T., et al. 2012, Sptz Prop, 90009

Bradley, L. D., Bouwens, R. J., Zitrin, A., et al. 2012a, ApJ, 747, 3

Bradley, L. D., Trenti, M., Oesch, P. A., et al. 2012b, ApJ, 760, 108

Brammer, G. B., van Dokkum, P. G., \& Coppi, P. 2008, ApJ, 686, 1503

Bruzual, G., \& Charlot, S. 2003, MNRAS, 344, 1000

Calzetti, D., Armus, L., Bohlin, R. C., et al. 2000, ApJ, 533, 682

Coe, D., Umetsu, K., Zitrin, A., et al. 2012, ApJ, 757, 22

Curtis-Lake, E., McLure, R. J., Dunlop, J. S., et al. 2013, MNRAS, 429, 302

Daddi, E., Dickinson, M., Morrison, G., et al. 2007, ApJ, 670, 156

Davé, R., Oppenheimer, B. D., \& Finlator, K. 2011, MNRAS, 415, 11

de Barros, S., Schaerer, D., \& Stark, D. P. 2012, A\&A, in press (arXiv:1207.3663)

Erb, D. K., Steidel, C. C., Shapley, A. E., et al. 2006, ApJ, 647, 128 Fumagalli, M., Patel, S. G., Franx, M., et al. 2012, ApJL, 757, L22

González, V., Bouwens, R., llingworth, G., et al. 2014, ApJ, 781, 34 González, V., Bouwens, R. J., Labbé, I., et al. 2012, ApJ, 755, 148

González, V., Labbé, I., Bouwens, R. J., et al. 2010, ApJ, 713, 115

Kennicutt, R. C., Jr. 1998, ARA\&A, 36, 189

Koekemoer, A. M., Faber, S. M., Ferguson, H. C., et al. 2011, ApJS, 197, 36

Kriek, M., van Dokkum, P. G., Labbé, I., et al. 2009, ApJ, 700, 221

Labbé, I., González, V., Bouwens, R. J., et al. 2010a, ApJL, 716, L103

Labbé, I., González, V., Bouwens, R. J., et al. 2010b, ApJL, 708, L26

Labbé, I., Oesch, P. A., Bouwens, R. J., et al. 2013, ApJL, 777, L19

Lorenzoni, S., Bunker, A. J., Wilkins, S. M., et al. 2011, MNRAS, 414, 1455

Makovoz, D., \& Khan, I. 2005, adass XIV, 347, 81

Meurer, G. R., Heckman, T. M., \& Calzetti, D. 1999, ApJ, 521, 64

Neistein, E., \& Dekel, A. 2008, MNRAS, 388, 1792

Noeske, K. G., Weiner, B. J., Faber, S. M., et al. 2007, ApJL, 660, L43

Oesch, P. A., Bouwens, R. J., Illingworth, G. D., et al. 2012, ApJ, 745, 110

Oesch, P. A., Bouwens, R. J., Illingworth, G. D., et al. 2013, ApJ, 773, 75

Oke, J. B., \& Gunn, J. E. 1983, ApJ, 266, 713

Ono, Y., Ouchi, M., Mobasher, B., et al. 2012, ApJ, 744, 83

Ouchi, M., Ellis, R., Ono, Y., et al. 2013, ApJ, 778, 102

Postman, M., Coe, D., Benítez, N., et al. 2012, ApJS, 199, 25

Reddy, N., Dickinson, M., Elbaz, D., et al. 2012, ApJ, 744, 154

Salpeter, E. E. 1955, ApJ, 121, 161

Schaerer, D., \& de Barros, S. 2009, A\&A, 502, 423

Schaerer, D., \& de Barros, S. 2010, A\&A, 515, A73

Schenker, M. A., Ellis, R. S., Konidaris, N. P., \& Stark, D. P. 2013a, ApJ, 777,67

Schenker, M. A., Robertson, B. E., Ellis, R. S., et al. 2013b, ApJ, 768, 196

Shim, H., Chary, R.-R., Dickinson, M., et al. 2011, ApJ, 738, 69

Stanway, E. R., Bunker, A. J., \& McMahon, R. G. 2003, MNRAS, 342, 439

Stark, D. P., Ellis, R. S., Bunker, A., et al. 2009, ApJ, 697, 1493

Stark, D. P., Schenker, M. A., Ellis, R., et al. 2013, ApJ, 763, 129

Steidel, C. C., Adelberger, K. L., Giavalisco, M., Dickinson, M., \& Pettini, M. 1999, ApJ, 519, 1

Tilvi, V., Papovich, C., Tran, K.-V. H., et al. 2013, ApJ, 768, 56

van der Wel, A., Straughn, A. N., Rix, H.-W., et al. 2011, ApJ, 742, 111

Weinmann, S. M., Neistein, E., \& Dekel, A. 2011, MNRAS, 417, 2737

Yabe, K., Ohta, K., Iwata, I., et al. 2009, ApJ, 693, 507

Zitrin, A., Broadhurst, T., Barkana, R., Rephaeli, Y., \& Benítez, N. 2011, MNRAS, 410, 1939

Zitrin, A., Broadhurst, T., Umetsu, K., et al. 2009, MNRAS, 396, 1985

Zitrin, A., Broadhurst, T., Umetsu, K., et al. 2010, MNRAS, 408, 1916 